$51.7 \mathrm{mcg} / \mathrm{dL} \mathrm{BPb}$. Subjects who went to practice 12 or more times a year reported a greater percentage of miscarriages in their partners (24\% vs 0\%). Twelve percent (8) showed an increase 35 in neuropsychiatric symptoms. The $\mathrm{BPb}$ multiple linear regression model explains $R^{2}=44.15 \%$, as follows: those who had $\geq 12$ practice sessions per year $\beta=0.5339$ and use of PbGC $\beta=0.3651$.

Conclusions Using firearms and PbGC contribute to increasing $\mathrm{BPb}$ in the studied personnel. The $\mathrm{BPb}$ concentrations found, despite being low, are a health risk, as evidenced by the prevalence of neuropsychiatric symptoms. The caretakers at the shooting range are at a higher risk.

\section{THE ASSOCIATION BETWEEN PARAOXONASE (PON1) POLYMORPHISMS AND CARDIOVASCULAR INJURIES AMONG NANOWORKERS}

${ }^{1} \mathrm{~S}$ Liou, ${ }^{2} \mathrm{Y}$ Chung, ${ }^{2} \mathrm{H}$ Liao, ${ }^{3} \mathrm{M}$ Lin, ${ }^{3} \mathrm{C}$ Lin, ${ }^{4} \mathrm{~W}$ Li, ${ }^{2} \mathrm{C}$ Wang, ${ }^{2} \mathrm{~W}$ Wu. ${ }^{1}$ National Health Research Institutes, Miaoli County, Taiwan; ${ }^{2}$ National Health Research Institutes, Miaoli, Taiwan; ${ }^{3}$ Institute of Occupational Safety and Health, Taipei, Taiwan; ${ }^{4}$ University of Washington, Seattle, United States of America

\subsection{6/oemed-2013-101717.10}

Objectives The market of nanomaterials products is rapidly expanding. The lack of scientific evidence describing accompanying exposure and cardiovascular health risks regarding its guidance and regulation. The objectives of this study was to examine (1) the association between different nanomaterials exposure and cardiovascular biomarkers and (2) effect of PON1 Q192R genetic polymorphism on cardiovascular biomarkers among nanoworkers. Methods In this cross-sectional study, we recruited 258 workers exposed to nanomaterials and 200 non-exposed controls from 14 nanomaterial handling plants in Taiwan from 2009 to 2011. The non-exposed controls were selected from the same plants, but they did not handle nanomaterials. We used the control banding matrix to categorize the risk level of nanoworkers. For each participant, we collected blood specimens to measure cardiovascular biomarkers and genotyping of PON1 (Q192R). In addition, heart rate variability (HRV) was tested.

Results PON1 Q192R genetic polymorphism associated with PON1 activity and HRV (SDNN, RMSSD, and TP). We also found that different nanomaterials exposure affected particular cardiovascular biomarkers by controlling PON1 Q192R genetic polymorphism. Such as nano-carbon tube exposure resulted in a decrease in fibrinogen. Nano- $\mathrm{SiO}_{2}$, nano-TiO ${ }_{2}$ and nano-Ag exposure separately resulted in an increase in fibrinogen, VCT and RMSSD.

Conclusion This study adopted comprehensive cardiovascular examinations to establish the association between different nanomaterials exposure and cardiovascular injuries. We also found possible causal associations that NPs may activate the autonomic nervous system and result in alterations of heart rate variability. Additionally, PON1 Q192R genetic polymorphisms were significant variables for PON1 activity and HRV.

\section{SECOND HAND TOBACCO SMOKE EXPOSURE AND CHANGES IN HEART RATE VARIABILITY AMONG CONSTRUCTION WORKERS}

'J Z Zhang, 'Fang, 'Mittleman, 'Chiristiani, ${ }^{2}$ Cavallari. ${ }^{1}$ Harvard School of Public Health, Boston, United States of America; ${ }^{2}$ University of Connecticut Health Center, Farmington, United States of America

10.1136/oemed-2013-101717.11
Objective Although it has been well defined that long-term exposure to secondhand smoke (SHS) is associated with cardiovascular mortality, the acute cardiovascular effects of SHS exposure are still being explored. The aim of this study was to examine cardiovascular autonomic response, as measured by heart rate variability (HRV), to short-term SHS exposure among boilermaker construction workers.

Methods Boilermakers were recruited from a local apprentice hall where they were monitored while exposed to SHS for approximately 6 hours. A repeated measures study design was used where resting 5-lead ECGs were taken from individuals before SHS exposure (baseline), following SHS exposure (post) and the morning following SHS exposure (next-morning). The ECG recording was analysed in time (SDNN, rMSSD) and frequency (LF, HF) domain parameters in 5-minute averages. Personal SHS exposure was monitored through SidepakTM Aerosol Monitor and quantified by fine particulate matter (PM2.5). Separate linear mixed-effects regression models were used to compare post- or next-morning after controlling for baseline. Exposure-response relationships with PM2.5 were examined.

Results 52 male boilermakers, including 30 non-smokers, were monitored between June 2010 and June 2012. The mean SHS exposure was $167 \mathrm{ug} / \mathrm{m}^{3}$. After SHS exposure post 5-minute SDNN, rMSSD, LF and HF were significantly $(\mathrm{p}<0.05)$ lower than before SHS exposure. We observed significant $(\mathrm{p}<0.05)$ differences in SDNN, rMSSD, LF and HF between smoker and non-smokers. However, this effect had no linear dose-response relationship with PM2.5. The next morning SDNN, rMSSD, LF and HF were also significantly $(\mathrm{p}<0.05)$ lower than the baseline, but no linear dose-response with PM2.5 was observed.

Conclusion We observed a change in HRV both immediately and the next morning following SHS exposure. However, results indicated that there was no linear dose-response relationship between PM2.5 and HRV. It is unclear whether SHS-derived PM2.5 or other factors contributed to the altered cardiovascular autonomic response.

\section{MORTALITY AND ACUTE MYOCARDIAL INFARCTION IN RCS EXPOSED WORKERS AT A SWEDISH PORCELAIN FACTORY}

${ }^{1} \mathrm{E}$ K Wiebert, ${ }^{2}$ Alderling, ${ }^{3}$ Svartengren, ${ }^{1}$ Gustavsson, ${ }^{1}$ Plato. ${ }^{1}$ Karolinska Institute, Stockholm, Sweden; ${ }^{2}$ Stockholm County Council, Stockholm, Sweden; ${ }^{3}$ Swedish Work Environment Authority, Stockholm, Sweden

\subsection{6/oemed-2013-101717.12}

Objectives Exposure to silica dust is a health hazard in the ceramic industry. The Swedish porcelain factory Gustavsberg started production in 1827, and have since 1971 performed occupational measurements of respirable crystalline silica dust (RCS). We have studied mortality and incidence of acute myocardial infarction (AMI) among workers at the factory.

Methods Exposure data from 436 personal measurements of RCS and respirable dust from 1971-2006 were processed and annual average of exposure levels were calculated. Exposure before 1971 was estimated. Analyses of mortality included 648 men and 315 women, employed for at least one year at the factory in 1958-2009. Forty three different causes of death were studied. Separate analyses of incidence of first time event of AMI included 358 men and 217 women.

Analyses were performed for all workers and for men and women separately. We also studied the effect of latency, duration and cumulative exposure to RCS and respirable dust. 
Results RCS-levels among highly exposed workers were five times higher than the OEL and ten times higher in the early 1970s compared with 2000. Workers exposed to RCS had an increased risk of mortality from respiratory diseases, SMR 1.75 (95\% CI 1.22-2.44). The risk was more pronounced in men, SMR 1.86 (1.22-2.70). Among women, mortality from diseases of the circulatory system was increased, although not statistically significant.

A non-significant increase in the incidence of AMI was also observed, with slightly higher point estimates for women than for men. No dose-response relationship was observed in any analysis. We observed eight cases of silicosis, and seven appeared with more than 30 years latency.

Conclusions The RCS-levels at the porcelain factory were well above the OEL in the 1970s. We found an increased mortality from respiratory diseases which can be attributed to the harmful effects of RCS on the lung.

\section{FACTORS ASSOCIATED WITH LONG-TERM SICKNESS ABSENCE DUE TO MENTAL AND BEHAVIOURAL DISORDERS}

J S Silva-Junior, Fischer. Public Health School, University of São Paulo, São Paulo, Brazil

10.1136/oemed-2013-101717.13

Objective To evaluate the factors associated with long term absences from work due to mental and behavioural disorders, including the interactions of two theoretical models of occupational stress.

Methods A case control study was conducted among workers seeking social security benefits in São Paulo, Brazil. They were absent from work more than fifteen days due to medical conditions, confirmed by social security medical doctors. Cases were workers diagnosed with mental and behavioural disorders. Controls were patients with clinical disability due to other medical problems. Both groups, 160 cases and 225 controls, were randomly selected in an agency of Brazilian Social Security Institution. They were interviewed and answered a comprehensive questionnaire about sociodemographic data, habits and lyfestyle, occupational information, perception of work conditions and clinical history. Multiple logistic regressions were performed to evaluate the hierarchical association of independent variables with the outcome.

Results Mental and behavioural disorders were associated with females (OR 2.48, 95\% CI 1.43- 4.30), self-reported skin colour as white (OR 2.16, 95\% CI 1.30-3.59), higher school education (OR 2.07, 95\% CI 1.15-3.71), high tobacco consumption (OR 5.10, 95\% CI 1.55-16.80), high alcohol intake (OR 8.91, 95\% CI 1.2662.90), employed by a public company (OR 3.33, 95\% CI 1.0110.97), exposure to violence at work (OR 4.23, 95\% CI 1.929.28), perception of high strain combined with low social support and effort-reward imbalance combined with high overcommitment at work (OR 5.70, 95\% CI 2.67-12.18), and more than two clinical disorders in the past year (OR 2.66, 95\% CI 1.59-4.43).

Conclusions There are variables in all hierarchical levels associated with long term sickness absence due to mental and behavioural disorders. Occupational stressors are strongly associated with these conditions. The public health policies must consider social security data to establish guidelines for health promotion in primary level, in order to minimise social and economic impacts.

\section{ASSOCIATIONS BETWEEN COMMUTING AND SOCIAL CAPITAL IN A CROSS-SECTIONAL POPULATION SURVEY IN SOUTHERN SWEDEN}

K M Mattisson, Kristina, Stroh, Håkansson. Lund University, Lund, Sweden

\subsection{6/oemed-2013-101717.14}

Objectives Commuting has been claimed to increase the material wealth. Through geographically expanding peoples labour markets, through increased commuting possibilities, more work opportunities occurs that will strengthen the economy. Although economy and flexibility to choose where to live and work will improve for the individual, several studies have shown that health become aggravated with increased commuting. One plausible explanation to the negative health is reduced social capital. Social capital is a resource emerging from social relations and the strength of those, on an individual and societal level. This resource can be used to solve problems of individual and collective character. Our primary hypothesis is that the longer the commuting time the less time for social participation and thereby a lower social capital.

Methods The study design is cross-sectional and the study material consists of two retrospective data sets collected through questionnaires in 2004 and 2008. The study population consist of respondents working more than $30 \mathrm{~h} /$ week, 18 to 65 years old and answering questions about commuting $(\mathrm{N}=21,088$ persons). Commuting is defined as transportation from the home to the work place, characterised by duration one way $(<30 \mathrm{~min}$, 30-60 min, >1 h) and mode (active, car, public). Social capital is defined as a combination of questions on social participation and generalised trust to other people. Relationships between social capital and the exposure variable will be investigated with log-binomial regression. Social participation and generalised trust to will also be investigated separately.

Results Has not yet been produced but a primary analysis done with logistic regression (as an indicator, instead of logbinomial regression) seems to show an association between social participation and increasing commuting time both with car and public, compared to active commuting. Low generalised trust seemed not to be strongly associated with commuting.

(Results will be presented on EPICOH 2013)

\section{REPRODUCTIVE HEALTH OUTCOME FROM CHEMICAL EXPOSURE IN A DRUG LABORATORY IN MALAYSIA}

C W Hoe. University of Malaya, Kuala Lumpur, Malaysia

\subsection{6/oemed-2013-101717.15}

Introduction Chemicals in the workplaces have been known to cause various health effects to the workers range from just irritation to causing cancers. A risk assessment was conducted in a hospital based dangerous drug monitoring laboratory in Kuala Lumpur, Malaysia.

Methods The method is a Qualitative Risk Assessment method using the combination techniques of Hazard Identification, Risk Assessment and Control (HIRAC) and the Job Safety Analysis (JSA). A questionnaire survey on the medical and health problem faced by the staff was also distributed during the survey.

Results The assessment found that four of the workers gave adverse reproductive health outcome; a 25 years old female worker having history of spontaneous abortion and was unable to conceive, a 51 years old female worker has two spontaneous 